Molecules 2004, 9, 957-967

molecules

ISSN 1420-3049

http://www.mdpi.org

\title{
New 1,2,3-Selenadiazole and 1,2,3-Thiadiazole Derivatives
}

\section{Mousa Al-Smadi * and Samer Ratrout}

Department of Applied Chemical Science, Jordan University of Science and Technology, P.O. Box 3030, Irbid 22110, Jordan

* Author to whom correspondence should be addressed; e-mail: mariam10@just.edu.jo

Received: 13 July 2004 / Accepted: 9 September 2004 / Published: 30 November 2004

\begin{abstract}
New 1,2,3-thiadiazole and 1,2,3-selenadiazole derivatives, 14-23, were prepared from the ketones 1-5 via the corresponding semicarbazones or hydrazones 6-12. The Hurd-Mori and Lalezari methods were used, respectively, for the preparation of these 1,2,3-thiadiazole and 1,2,3-selenadiazole derivatives. The intermediate $\mathbf{1 3}$ was also trapped, separated and fully characterized. These derivatives are important for photocrosslinking processes and due to their potential biological activity.
\end{abstract}

Keywords: Semicarbazone, hydrazone, ferrocene, 1,2,3-selenadiazole, 1,2,3-thiadiazole.

\section{Introduction:}

Interest in the synthesis of selenium and sulfur containing compounds and the further utilization of these compounds in organic synthesis has been steadily increasing recently [1]. Particular interest in 1,2,3-selenadiazole and 1,2,3-thiadiazole derivatives stems from the fact that they can undergo a wide variety of reactions where they act as 1,3-dipoles or as a source of selenium or sulfur and hence they have attracted much attention for the synthesis of different organoselenium and organosulfur compounds [2] in both the acyclic and cyclic series [3]. In spite of the obvious attraction of Se and Sheterocycles, only a few preparative routes have been described. Lalezari et al. [4-6] were the first to report the synthesis of a 1,2,3-selenadiazole ring by analogy with the 1,2,3-thiadiazole system, which had been prepared previously by Hurd and Mori [7]. We report herein the synthesis of new compounds containing 1,2,3-selenadiazole and 1,2,3-thiadiazole rings using the Lalezari et al. and Hurd and Mori methods. 


\section{Results and Discussion}

Our synthetic procedure for new 1,2,3-selenadiazole and 1,2,3-thiadiazole derivatives started from a variety of ketones 1-5 containing $\alpha$-methylene groups (Scheme 1) that were first converted into their corresponding tosyl or acyl hydrazones or semicarbazones and then further converted into 1,2,3selenadiazole ring derivatives by the selenium dioxide oxidative ring closure of these semicarbazone or hydrazone derivatives [8-11] and into 1,2,3-thiadiazoles by reaction of the hydrazones or semicarbazones with thionyl chloride [12-16].

Scheme 1. Ketones used in the preparation of new 1,2,3-thiadiazole and 1,2,3selenadiazole compounds.

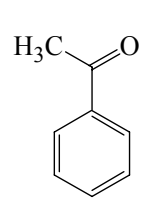

(1)

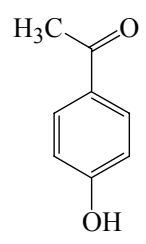

(2)

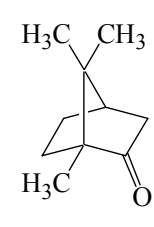

(3)

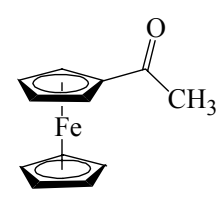

(4)

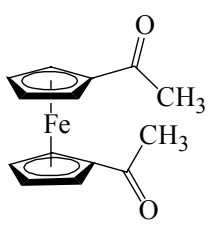

(5)

The general equations for the preparation of 1,2,3-selenadiazole and 1,2,3-thiadiazole derivatives are shown in Scheme 2.

\section{Scheme 2}

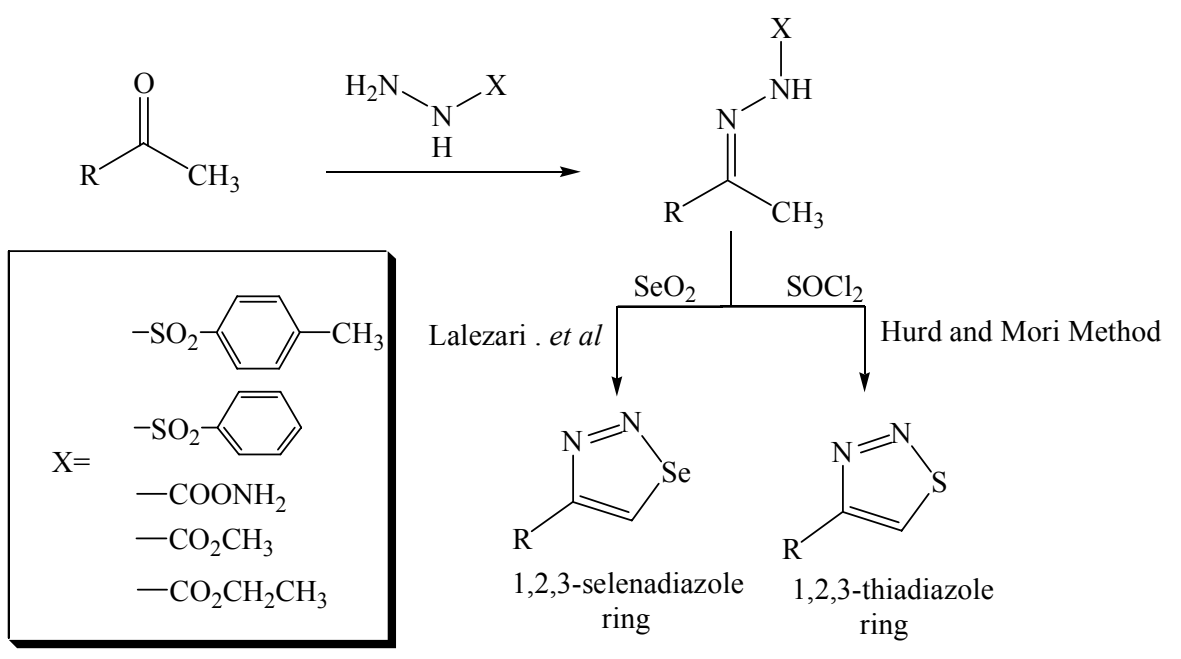

Table 1 shows the structures of the newly prepared compounds, melting point ranges and the percentage yields of these compounds. 
Table 1.

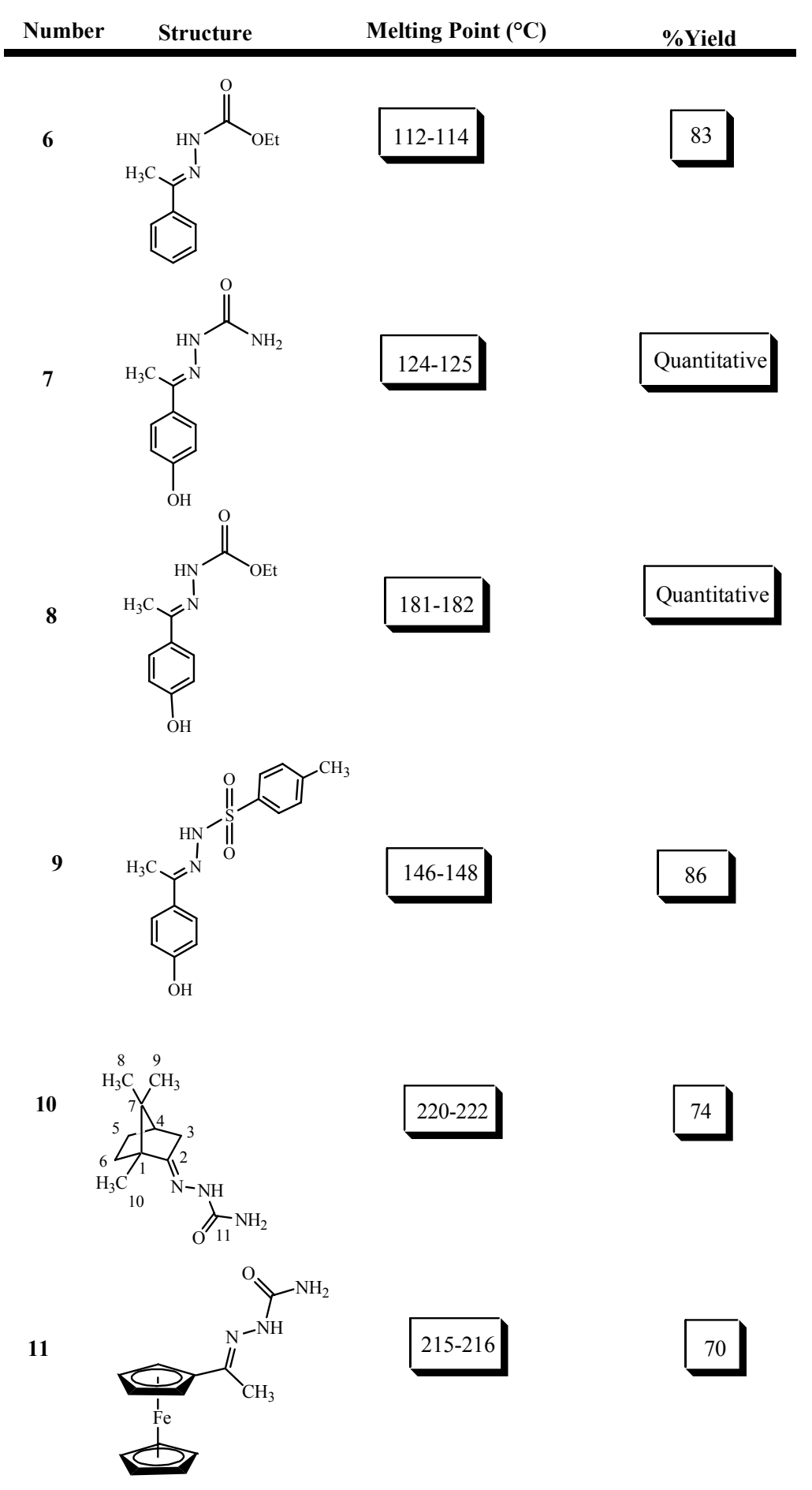


Table 1. Cont.

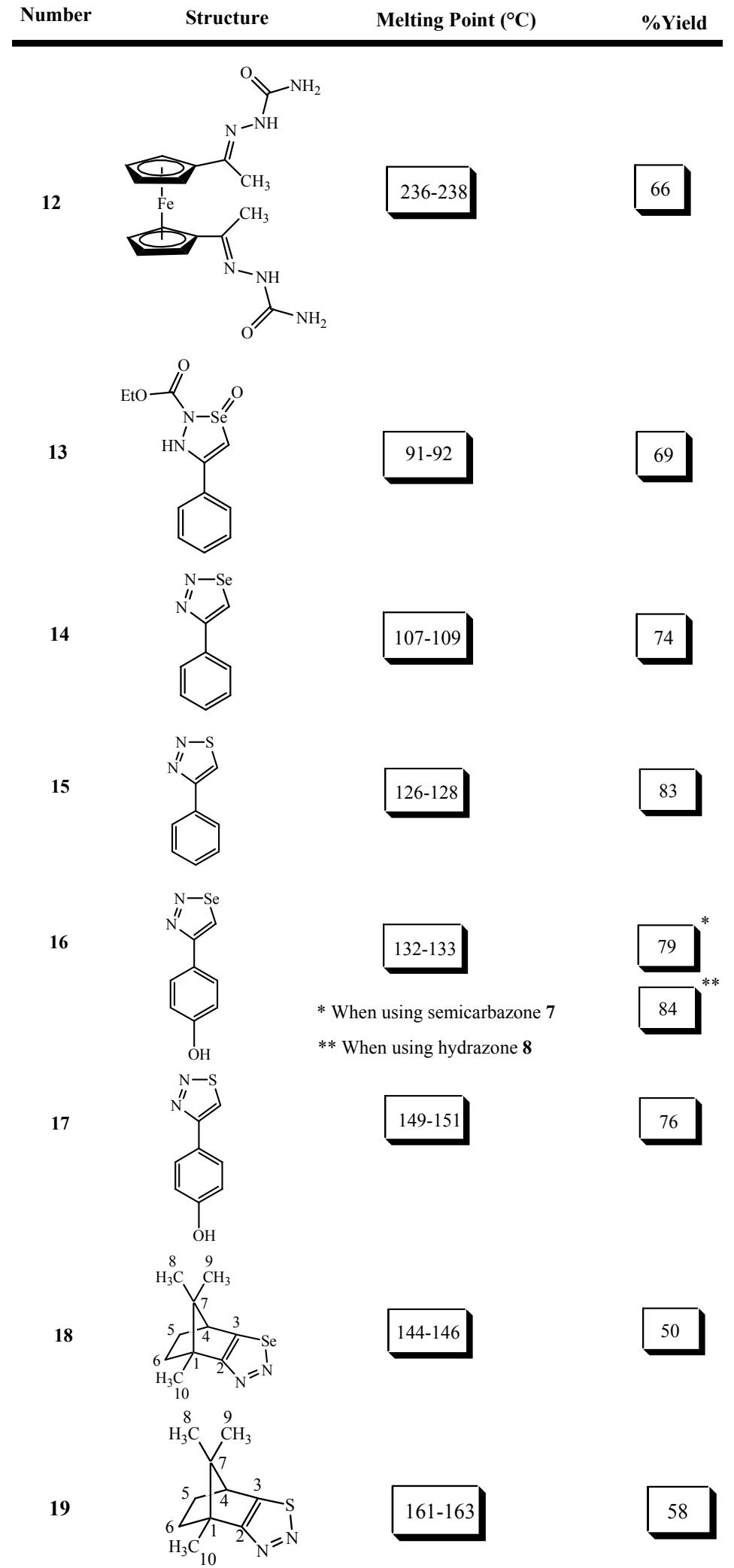


Table 1. Cont.

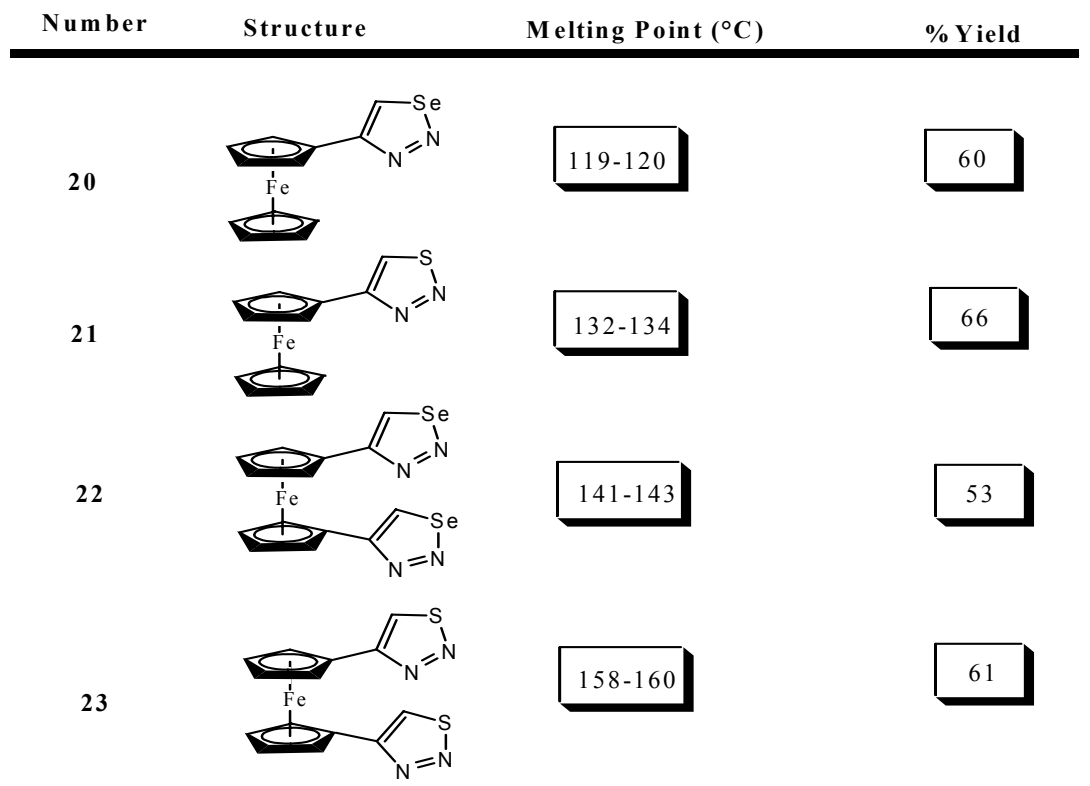

Compound 13 was of particular interest as it represents the first isolable and stable intermediate in the mechanism for 1,2,3-selenadiazole ring cyclization. Scheme 3 shows the proposed mechanism for the formation of compound 13. Its structure was confirmed by IR, ${ }^{1} \mathrm{H}-\mathrm{NMR},{ }^{13} \mathrm{C}-\mathrm{NMR}$, mass spectrometry and elemental analysis.

Scheme 3. Mechanism for the formation of compound $\mathbf{1 3}$
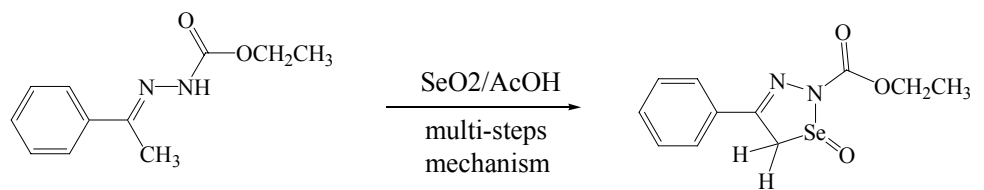

The first intermediate

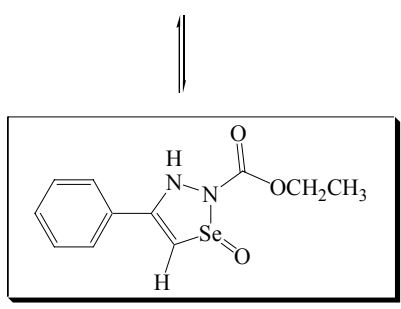

The second intermediate Compound 13

\section{Acknowledgements}

We are grateful to the Department of Applied Chemistry-Deanship of Scientific Research - Jordan University of Science and Technology for financial support. Also, we thank Prof. H. Meier of Mainz University - Germany for helpful and valuable discussions. 


\section{Experimental}

\section{General}

The solvents used were purified by standard procedures. The melting points (m.p) were determined on an Electrothermal digital melting point apparatus and uncorrected. Infrared (IR) spectra of pure substances were recorded for $\mathrm{KBr}$ pellets using a NICOLET $410 \mathrm{FT}$-IR spectrometer $\left(v\right.$ in $\left.\mathrm{cm}^{-1}\right)$. The ${ }^{1} \mathrm{H}$ - and ${ }^{13} \mathrm{C}-\mathrm{NMR}$ spectra were recorded on Bruker AM 400 and $\mathrm{AC} 200$ spectrometers in $\mathrm{CDCl}_{3}$ or DMSO- $\mathrm{d}_{6}$. The spectral data are reported in delta $(\delta)$ units relative to the TMS reference peak. The mass spectra were registered using MAT CH7A (Varian, EI: 70eV Ionizing energy, electron ionization) and MAT95 (Finnigan, FD: 5kV Ionizing energy, field desorption) instruments. The signals are given as $\mathrm{m} / \mathrm{z}$ with the relative intensity between brackets. Elemental analyses were performed in the analytical laboratory of the Institute of Organic Chemistry of University of Mainz, Germany. D-(+)-camphor, acetyl ferrocene, diacetyl ferrocene, p-hydroxyacetophenone, acetophenone, ethyl hydrazine carboxylate, semicarbazide hydrochloride and sodium acetate were obtained from Aldrich.

General procedure for the preparation of semicarbazones 7, 10, 11 and 12.

A mixture of semicarbazide hydrochloride (1.00 equivalent) and sodium acetate (1.00 equivalent) was dissolved in absolute ethanol $(45 \mathrm{~mL})$. The mixture was heated for 15 min under reflux, then filtered while hot to remove precipitated sodium chloride. The filtrate was then mixed with $p$-hydroxyacetophenone (2, 0.95 equivalents), $\mathrm{D}$-(+)-camphor (3, 0.95 equivalents), acetyl ferrocene (4, 0.95 equivalents) or diacetyl ferrocene $(5,0.45$ equivalents), respectively, and the resulting mixtures were heated to reflux, a few drops of concentrated hydrochloric acid were added and heating under reflux with continuous removal of the generated water was continued overnight. The solvent was removed under vacuum and the residue was washed with diethyl ether or chloroform.

$N^{\prime}$-[1-(4-Hydroxyphenyl)-ethylidene] semicarbazone (7). White solid powder, yield = quantitative; IR: $v$ 3448, 3220, 1667, 1572, 1411, $1351 \mathrm{~cm}^{-1} ;{ }^{1} \mathrm{H}-\mathrm{NMR}$ (DMSO-d $\left.{ }_{6}\right): \delta 2.16\left(\mathrm{~s}, 3 \mathrm{H}, \mathrm{CH}_{3}\right), 6.98 / 7.65$ (AA'BB', 4H, aromatic H), $9.97(\mathrm{~s}, 1 \mathrm{H}, \mathrm{N}-\mathrm{H}) ;{ }^{13} \mathrm{C}-\mathrm{NMR}$ (DMSO-d $)$ ): $\delta 14.2\left(\mathrm{CH}_{3}\right), 114.8 / 127.6(\mathrm{CH}$, phenylene), 131.3/158.7 ( $\mathrm{C}_{\mathrm{q}}$, phenylene), $148.9(\mathrm{CN}), 154.3(\mathrm{CO}) ; \mathrm{MS}(\mathrm{m} / \mathrm{z}, \%): 193\left(\mathrm{M}^{+}, 100 \%\right)$; Anal. Calcd. for $\mathrm{C}_{9} \mathrm{H}_{11} \mathrm{~N}_{3} \mathrm{O}_{2}$ : C, 55.95; H, 5.74; N, 21.75. Found: C, 55.91; H, 5.68; N, 21.81 .

$N^{\prime}$-(1,7,7-Trimethylbicyclo[2.2.1] hept-2-ylidene) semicarbazone (10). Pale white-green solid, yield = 74\%; IR: v 3462, 3199, 2949, 1688, 1585, $1489 \mathrm{~cm}^{-1}$; ${ }^{1} \mathrm{H}-\mathrm{NMR}$ (DMSO-d ${ }_{6}$ ): $\delta 0.65$ (s, 3H, $\left.\mathrm{C}_{10}\right), 0.85$ (s, 3H at $\left.\mathrm{C}_{9}\right), 0.91\left(\mathrm{~s}, 3 \mathrm{H}\right.$ at $\left.\mathrm{C}_{8}\right), 1.23 / 1.72\left(2 \mathrm{~m}, 4 \mathrm{H}\right.$ at $\left.\mathrm{C}_{5}, \mathrm{C}_{6}\right), 1.89\left(\mathrm{~d}, 2 \mathrm{H}, \mathrm{C}_{3}\right), 2.28\left(\mathrm{~m}, 1 \mathrm{H}\right.$ at $\left.\mathrm{C}_{4}\right), 5.14$ $\left(\mathrm{s}, 2 \mathrm{H}, \mathrm{NH}_{2}\right), 8.76$ (s, 1H, N-H); ${ }^{13} \mathrm{C}-\mathrm{NMR}\left(\mathrm{DMSO}-\mathrm{d}_{6}\right): \delta 54.84\left(\mathrm{C}_{1}\right), 157.48\left(\mathrm{C}_{2}\right), 34.18\left(\mathrm{C}_{3}\right), 43.47$ $\left(\mathrm{C}_{4}\right), 26.99\left(\mathrm{C}_{5}\right), 32.56\left(\mathrm{C}_{6}\right), 47.53\left(\mathrm{C}_{7}\right), 19.32\left(\mathrm{C}_{8}\right), 18.62\left(\mathrm{C}_{9}\right), 11.34\left(\mathrm{C}_{10}\right)$ and $161.74\left(\mathrm{C}_{11}\right) ; \mathrm{MS}(\mathrm{m} / \mathrm{z}$, \%): $209\left(\mathrm{M}^{+}, 100 \%\right)$; Anal. Calcd. for $\mathrm{C}_{11} \mathrm{H}_{19} \mathrm{~N}_{3} \mathrm{O}$ : C, 63.13; H, 9.15; N, 20.08. Found: C, 63.05; H, 9.10; N, 19.90. 
Acetyl ferrocene semicarbazone (11). Reddish orange solid, yield $=70 \%$ yield; IR: $v 3468,3192$, 3096, 1700, 1572, $1431 \mathrm{~cm}^{-1} ;{ }^{1} \mathrm{H}-\mathrm{NMR}\left(\mathrm{CDCl}_{3}\right): \delta 2.11\left(\mathrm{~s}, 3 \mathrm{H}, \mathrm{CH}_{3}-\mathrm{C}=\mathrm{N}\right), 4.13(\mathrm{~s}, 5 \mathrm{H}$, unsubstituted cyclopentadienyl ring), 4.52 and $4.30(2 \mathrm{~d}, 4 \mathrm{H}$, monosubstituted cyclopentadienyl ring), 8.38 (s, $1 \mathrm{H}, \mathrm{N}-$ $\mathrm{H}) ;{ }^{13} \mathrm{C}-\mathrm{NMR}\left(\mathrm{CDCl}_{3}\right): \delta 14.14\left(\underline{\mathrm{CH}}_{3}-\mathrm{C}=\mathrm{N}\right), 147.20(\underline{\mathrm{C}}=\mathrm{N}), 157.54(\mathrm{~N}-\underline{\mathrm{C}}(\mathrm{O})-\mathrm{N}), 69.15,69.80,66.65$, 83.44 (10C, cyclopentadienyl rings); MS (m/z, \%) : $285\left(\mathrm{M}^{+}, 100 \%\right)$; Anal. Calcd. for $\mathrm{C}_{13} \mathrm{H}_{15} \mathrm{~N}_{3} \mathrm{OFe}$ : C, 54.57; H, 5.64; N, 14.69. Found: C, 54.39; H, 5.54; N, 14.52 .

Diacetylferrocene semicarbazone (12). Dark reddish solid, yield $=68 \%$ yield; IR: $v$ 3456, 3191, 1712 , 1568, $1436 \mathrm{~cm}^{-1} ;{ }^{1} \mathrm{H}-\mathrm{NMR}\left(\mathrm{CDCl}_{3}\right): \delta 2.14\left(\mathrm{~s}, 6 \mathrm{H}, \mathrm{CH}_{3}-\mathrm{C}=\mathrm{N}\right), 4.59$ and $4.37(2 \mathrm{~d}, 8 \mathrm{H}$, monosubstituted cyclopentadienyl rings), $8.32(\mathrm{~s}, 2 \mathrm{H}, \mathrm{N}-\mathrm{H}) ;{ }^{13} \mathrm{C}-\mathrm{NMR}\left(\mathrm{CDCl}_{3}\right): \delta 14.28\left(2 \mathrm{C}, \mathrm{CH}_{3}-\mathrm{C}=\mathrm{N}\right), 147.39(2 \mathrm{C}$, $\underline{\mathrm{C}}=\mathrm{N}), 156.97$ (2C, N- $\underline{\mathrm{C}}(\mathrm{O})-\mathrm{N}), 69.16,69.83,83.24$ (10C, cyclopentadienyl rings); MS (m/z, \%) : 384 $\left(\mathrm{M}^{+}, 100 \%\right)$; Anal. Calcd. for $\mathrm{C}_{16} \mathrm{H}_{20} \mathrm{~N}_{6} \mathrm{O}_{2} \mathrm{Fe}: \mathrm{C}, 50.02 ; \mathrm{H}, 5.25 ; \mathrm{N}, 21.87$. Found: $\mathrm{C}, 50.08 ; \mathrm{H}, 5.21 ; \mathrm{N}$, 21.79 .

\section{General procedure for the preparation of hydrazones $\mathbf{6}$ and $\mathbf{8}$}

A mixture of acetophenone (1, 1.00 equivalent) or 4-hydroxyacetophenone (2, 1.00 equivalent) and ethyl hydrazine carboxylate (1.20 equivalents) was dissolved in dry, hot chloroform ( $80 \mathrm{~mL})$. When the reaction mixture started refluxing, two drops of concentrated hydrochloric acid were added and the mixture was then refluxed overnight with continuous removal of the water generated. The solvent was removed under vacuum and the residue was washed several times with diethyl ether or chloroform to remove excess reactants.

N'-[1-phenylethylidene]hydrazine carboxylic acid ethyl ester (6). White solid powder, yield = 83\%; IR: v 3192, 3051, 2975, 1732, 1540, $1035 \mathrm{~cm}^{-1}$; ${ }^{1} \mathrm{H}-\mathrm{NMR}$ (DMSO-d (D) $\delta 1.24\left(\mathrm{t}, 3 \mathrm{H}, \mathrm{CH}_{2} \mathrm{CH}_{3}\right), 2.19$ (s, 3H, $\left.\mathrm{CH}_{3}-\mathrm{C}=\mathrm{N}\right), 4.14$ (q, $2 \mathrm{H}, \mathrm{CH}_{2} \mathrm{CH}_{3}$ ), 7.27-7.33 (m, 3H, aromatic), 7.69-7.71 (m, 2H, aromatic), $10.09(\mathrm{~s}, \quad 1 \mathrm{H}, \quad \mathrm{N}-\mathrm{H}) ;{ }^{13} \mathrm{C}-\mathrm{NMR}\left(\mathrm{DMSO}-\mathrm{d}_{6}\right): \delta 13.93 \quad\left(\mathrm{OCH}_{2} \underline{\mathrm{CH}_{3}}\right), 14.71 \quad\left(\underline{\mathrm{CH}}_{3}-\mathrm{C}=\mathrm{N}\right), 60.61$ $\left(\mathrm{O}^{-} H_{2} \mathrm{CH}_{3}\right), 148.93(\underline{\mathrm{C}}=\mathrm{N}), 154.34(\mathrm{O}-\underline{\mathrm{C}}(\mathrm{O})-\mathrm{N}), 126.11,128.32,128.89$ and 138.47 (aromatic carbons); $\mathrm{MS}(\mathrm{m} / \mathrm{z}, \%): 206\left(\mathrm{M}^{+}, 100 \%\right)$; Anal. Calcd. for $\mathrm{C}_{11} \mathrm{H}_{14} \mathrm{~N}_{2} \mathrm{O}_{2}$ : C, 64.06; H, 6.84; N, 13.58 . Found: C, 63.70; H, 6.79; N, 13.60 .

N'-[1-(hydroxyphenyl)ethylidene]hydrazine carboxylic acid ethyl ester (8). White solid powder, yield $=$ quantitative; IR: $v 3385,3263,1726,1610,1501,1406 \mathrm{~cm}^{-1} ;{ }^{1} \mathrm{H}-\mathrm{NMR}\left(\mathrm{DMSO}-\mathrm{d}_{6}\right): \delta 1.28(\mathrm{t}, 3 \mathrm{H}$, $\mathrm{CH}_{2} \mathrm{CH}_{3}$ ), 2.21 (s, 3H, $\mathrm{CH}_{3}-\mathrm{C}=\mathrm{N}$ ), 4.16 (q, 2H, $\mathrm{CH}_{2} \mathrm{CH}_{3}$ ), 7.28/7.70 (AA'BB', 4H, aromatic), 9.97 (s, $1 \mathrm{H}, \mathrm{N}-\mathrm{H}) ;{ }^{13} \mathrm{C}-\mathrm{NMR}\left(\mathrm{DMSO}-\mathrm{d}_{6}\right): \delta 13.97\left(\mathrm{OCH}_{2} \underline{\mathrm{CH}_{3}}\right), 14.77\left(\underline{\mathrm{CH}}_{3}-\mathrm{C}=\mathrm{N}\right), 60.58\left(\mathrm{OCH}_{2} \mathrm{CH}_{3}\right), 148.94$ $(\underline{\mathrm{C}}=\mathrm{N}), 154.47(\mathrm{O}-\underline{\mathrm{C}}(\mathrm{O})-\mathrm{N}), 114.3 / 127.9(\mathrm{CH}$, phenylene), 131.2/158.9 (Cq, phenylene); $\mathrm{MS}(\mathrm{m} / \mathrm{z}, \%)$ : $222\left(\mathrm{M}^{+}, 100 \%\right)$; Anal. Calcd. for $\mathrm{C}_{11} \mathrm{H}_{14} \mathrm{~N}_{2} \mathrm{O}_{3}$ : C, 59.44; H, 6.34; N, 12.60. Found: C, 59.49; H, 6.40; $\mathrm{N}, 12.66$.

N'-[1-(hydroxyphenyl)ethylidene] hydrazine tosylate (9).

Acetophenone $(2.50 \mathrm{mmol})$ was added to a warm solution of $p$-toluenesulfonic acid hydrazide $(2.70 \mathrm{mmol})$ in dry ethanol $(30 \mathrm{~mL})$. The solution was refluxed for $1.0 \mathrm{hr}$ and then concentrated to one-half of its original volume. After cooling the reaction mixture to room temperature, the product precipitated as a colorless powder which was washed with a small amount of cooled ethanol and dried 
to afford the title compound in $86 \%$ yield; IR: $v 3385,3263,1726,1610,1501,1406 \mathrm{~cm}^{-1} ;{ }^{1} \mathrm{H}-\mathrm{NMR}$ (DMSO-d $\left.{ }_{6}\right): \delta 2.21 / 2.38\left(2 \mathrm{~s}, 6 \mathrm{H}, \mathrm{CH}_{3}\right), 6.84 / 7.73\left(\mathrm{AA}^{\prime} \mathrm{BB}^{\prime}, 4 \mathrm{H}\right.$, aromatic), 7.37/7.54 (AA'BB', 8H, toluene), $10.31(\mathrm{~s}, 1 \mathrm{H}, \mathrm{N}-\mathrm{H}) ;{ }^{13} \mathrm{C}-\mathrm{NMR}\left(\mathrm{DMSO}_{\mathrm{d}}\right): \delta 14.19\left(\mathrm{CH}_{3}\right), 20.72\left(\mathrm{CH}_{3}\right.$, toluene), 114.17/127.63 (CH, phenylene), 130.51/159.80 (Cq, phenylene), 127.71/129.83 ( $\mathrm{CH}$, toluene), 136.58/143.29 (Cq, toluene), $152.64(\underline{\mathrm{C}}=\mathrm{N})$; $\mathrm{MS}(\mathrm{m} / \mathrm{z}, \%): 304\left(\mathrm{M}^{+}, 100 \%\right)$; Anal. Calcd. for $\mathrm{C}_{15} \mathrm{H}_{16} \mathrm{~N}_{2} \mathrm{O}_{3} \mathrm{~S}$ : C, 59.19; H, 5.30; N, 9.24; S, 10.53. Found: C, 59.22; H, 5.26; N, 9.31; S, 10.59 .

\section{General procedure for the preparation of 1,2,3-selenadiazole derivatives 13, 15, 17, 19, 21}

Each of the semicarbazones $7(1.98 \mathrm{mmol}), \mathbf{1 0}(1.68 \mathrm{mmol}), \mathbf{1 1}(0.29 \mathrm{mmol})$ or $\mathbf{1 2}(1.00 \mathrm{mmol})$ or the hydrazones 6 or $8(2.50 \mathrm{mmol})$, respectively, was dissolved in glacial acetic acid $(40 \mathrm{~mL})$ with vigorous stirring and gentle heating to $40-45^{\circ} \mathrm{C}$ (in case of the hydrazone 6 , the solution was stirred at room temperature). The solution was treated with selenium dioxide powder $(2.70 \mathrm{mmol}, 3.60 \mathrm{mmol}$, $0.57 \mathrm{mmol}, 4.00 \mathrm{mmol}$ or $2.72 \mathrm{mmol}$, respectively) and the mixture was kept under vigorous stirring. After ca. 2 min the color of the mixture becomes red. Monitoring of the reaction by TLC (eluent: 1:4 ethyl acetate-hexane) showed that the reaction was complete in $24 \mathrm{hr}$. Thes mixture were filtered and the filtrates poured into ice water and extracted with $\mathrm{CDCl}_{3}(3 \times 50 \mathrm{~mL})$. The combined organic layers were washed with saturated sodium hydrogen carbonate solution. dried over magnesium sulphate and the solvent was removed under vacuum to afford the crude title compounds, which were further purifed as indicated under each heading.

Ethyl-3,5-dihydro-4-phenyl-1-oxo-1,2,3-selenadiazole-2-carboxylate (13). The residue was washed with hexane and the insoluble solid was chromatographed using 1:3 ethyl acetate-hexane as eluent to give compound $\mathbf{1 3}$ as a pale yellow solid in 69\% yield; IR: $v$ 3206, 2982, 1739, 1694, 1598, 1515, 1226, $726 \mathrm{~cm}^{-1}$; ${ }^{1} \mathrm{H}-\mathrm{NMR}\left(\mathrm{CDCl}_{3}\right): \delta 1.29\left(\mathrm{t}, 3 \mathrm{H}, \mathrm{CH}_{2} \mathrm{CH}_{3}\right), 4.30\left(\mathrm{q}, 2 \mathrm{H}, \mathrm{CH}_{2} \mathrm{CH}_{3}\right), 7.20-7.22(\mathrm{~m}, 3 \mathrm{H}$, aromatic), $7.49-7.51\left(\mathrm{~m}, 2 \mathrm{H}\right.$, aromatic), $8.62(\mathrm{~s}, 1 \mathrm{H}, \mathrm{N}-\mathrm{H}), 9.71\left(\mathrm{~s}, 1 \mathrm{H}\right.$, proton at $\mathrm{C}_{5}$ of selenadiazole ring); ${ }^{13} \mathrm{C}-\mathrm{NMR}\left(\mathrm{CDCl}_{3}\right): \delta 14.45\left(\mathrm{OCH}_{2} \underline{\mathrm{CH}} 3\right), 63.19\left(\mathrm{OCH}_{2} \mathrm{CH}_{3}\right), 126.29,126.85,128.35$ and 129.78 for benzene carbons, 130.77 and 149.50 for $\mathrm{C}_{4}$ and $\mathrm{C}_{5}$ carbons of the 1,2,3-selenadiazole ring, 152.48 for O- $\underline{\mathrm{C}}(\mathrm{O})-\mathrm{N}$; $\mathrm{MS}(\mathrm{m} / \mathrm{z}, \%): 283\left(\mathrm{M}^{+}, 100 \%\right)$; Anal. Calcd. For $\mathrm{C}_{11} \mathrm{H}_{12} \mathrm{O}_{3} \mathrm{~N}_{2} \mathrm{Se}: \mathrm{C}, 46.64 ; \mathrm{H}, 4.24 ; \mathrm{N}$, 9.89. Found: C, 46.53; H, 4.12; N, 9.79 .

4-Phenyl-[1,2,3]-selenadiazole (14). The residue was chromatographed using 1:4 ethyl acetate-hexane as mobile phase to give a yellow solid of compound $\mathbf{1 4}$ in 74\% yield; IR: $v$ 3061, 1687, 1591, 1483, $1235 \mathrm{~cm}^{-1} ;{ }^{1} \mathrm{H}-\mathrm{NMR}\left(\mathrm{CDCl}_{3}\right): \delta$ 7.29-7.33 (m, 3H, aromatic), 7.54-7.56 (m, 2H, aromatic), $8.93(\mathrm{~s}, 1 \mathrm{H}$, selenadiazole ring); ${ }^{13} \mathrm{C}-\mathrm{NMR}\left(\mathrm{CDCl}_{3}\right): \delta 126.41,127.62,127.90$ and 130.73 for benzene carbons, 162.53 and 133.41 for $\mathrm{C} 4$ and $\mathrm{C} 5$ carbons of the selenadiazole ring); $\mathrm{MS}(\mathrm{m} / \mathrm{z}, \%): 209\left(\mathrm{M}^{+}, 100 \%\right)$; Anal. Calcd. For $\mathrm{C}_{8} \mathrm{H}_{6} \mathrm{~N}_{2} \mathrm{Se}: \mathrm{C}, 45.95 ; \mathrm{H}, 2.89 ; \mathrm{N}, 13.40$. Found: C, 45.78; H, 2.93; N, 13.49.

4-[1,2,3]-Selenadiazole-4-yl-phenol (16). The residue was recrystalized from acetone/hexane to give a faint gray solid of compound $\mathbf{1 6}$ in 79\% yield (when semicarbazone 7 is used) and 84\% yield (when hydrazone 8 is used); IR: $v$ 3429, 3077, 1604, 1527, 1468, 1245, $803 \mathrm{~cm}^{-1} ;{ }^{1} \mathrm{H}-\mathrm{NMR}\left(\mathrm{CDCl}_{3}\right): \delta 2.10$ (s, 1H, OH group), 6.90/7.90 (AA'BB', 4H, phenylene), $9.20\left(\mathrm{~s}, 1 \mathrm{H}\right.$, selenadiazole ring); ${ }^{13} \mathrm{C}-\mathrm{NMR}$ 
$\left(\mathrm{CDCl}_{3}\right): \delta$ 114.81/126.40 (CH, phenylene), 129.82/158.70 (Cq, phenylene), 162.41/133.29 (C4/C5, selenadiazole ring carbons); MS (m/z, \%): $225\left(\mathrm{M}^{+}, 100 \%\right)$; Anal. Calcd. For $\mathrm{C}_{8} \mathrm{H}_{6} \mathrm{~N}_{2} \mathrm{OSe}$ : C, 42.69; H, 2.69; N, 12.44. Found: C, 42.74; H, 2.63; N, 12.48 .

7,10,10-Trimethyl-3-selena-4,5-diaza-tricyclo[5.2.1.0 $\left.0^{2,6}\right]$ deca-2(6),4-diene (18). The crude product was chromatograhed using 1:4 ethyl acetate-hexane as mobile phase to give a pale yellow-brown solid of compound 18 in 50\% yield; IR: $v 2949,1540,1420,1232 \mathrm{~cm}^{-1} ;{ }^{1} \mathrm{H}-\mathrm{NMR}\left(\mathrm{CDCl}_{3}\right): \delta 0.72(\mathrm{~s}, 3 \mathrm{H}$ at $\left.\mathrm{C}_{9}\right), 0.89$ (s, 3H at $\left.\mathrm{C}_{10}\right), 1.05\left(\mathrm{~s}, 3 \mathrm{H}\right.$ at $\left.\mathrm{C}_{8}\right), 1.44 / 1.82\left(2 \mathrm{~m}, 4 \mathrm{H}\right.$ at $\left.\mathrm{C}_{5}, \mathrm{C}_{6}\right), 2.29\left(\mathrm{~m}, 1 \mathrm{H}\right.$ at $\left.\mathrm{C}_{4}\right)$; ${ }^{13} \mathrm{C}-\mathrm{NMR}$ $\left(\mathrm{CDCl}_{3}\right): \delta\left(61.57, \mathrm{C}_{1}\right),\left(166.52, \mathrm{C}_{2}\right),\left(153.80, \mathrm{C}_{3}\right),\left(43.92, \mathrm{C}_{4}\right),\left(27.28, \mathrm{C}_{5}\right),\left(32.41, \mathrm{C}_{6}\right),\left(48.05, \mathrm{C}_{7}\right)$, $\left(19.52, \mathrm{C}_{8}\right),\left(18.59, \mathrm{C}_{9}\right),\left(11.09, \mathrm{C}_{10}\right) ; \mathrm{MS}(\mathrm{m} / \mathrm{z}, \%): 238\left(\mathrm{M}^{+}, 100 \%\right)$; Anal. Calcd. For $\mathrm{C}_{10} \mathrm{H}_{14} \mathrm{~N}_{2} \mathrm{Se}: \mathrm{C}$, 49.80; H, 5.85; N, 11.61. Found C, 49.71; H, 5.84; N, 11.60 .

[1,2,3]-Selenadiazole-4-yl-ferrocene (20). The crude product was purified as indicated for compound $\mathbf{1 8}$ to give an orange solid of compound $\mathbf{2 0}$ in $60 \%$ yield; IR: $v$ 3083, 1700, 1528, 1400, 1258, 816 $\mathrm{cm}^{-1} ;{ }^{1} \mathrm{H}-\mathrm{NMR}\left(\mathrm{CDCl}_{3}\right): \delta 8.89\left(\mathrm{~s}, 1 \mathrm{H}\right.$, proton at $\mathrm{C}_{5}$ selenadiazole ring), $4.17(\mathrm{~s}, 5 \mathrm{H}$, unsubstituted cyclopentadienyl ring), 4.47/5.00 (2d, 4H, monosubstituted cyclopentadienyl ring); ${ }^{13} \mathrm{C}-\mathrm{NMR}$ $\left(\mathrm{CDCl}_{3}\right): \delta 162.52$ and 133.28 for $\mathrm{C}_{4}$ and $\mathrm{C}_{5}$ selenadiazole ring, 68.04, 69.45, 69.71 and 85.00 for the carbons of the mono and unsubstituted cyclopentadienyl rings; MS (m/z, \%): $318\left(\mathrm{M}^{+}, 100 \%\right)$; Anal. Calcd. For $\mathrm{C}_{12} \mathrm{H}_{10} \mathrm{~N}_{2} \mathrm{SeFe}$ : C, 45.31; H, 3.49; N, 8.81. Found: C, 45.22; H, 3.47; N, 8.75.

Di-[1,2,3]-selenadiazole-4-yl-ferrocene (22). The crude product was chromatographed like compound $\mathbf{1 8}$ to give an orange solid of compound 22 in 64\% yield; IR: $v$ 3075, 1693, 1528, 1403, 1248, 821 $\mathrm{cm}^{-1}$; ${ }^{1} \mathrm{H}-\mathrm{NMR}\left(\mathrm{CDCl}_{3}\right): \delta 8.91(\mathrm{~s}, 2 \mathrm{H}$, selenadiazole rings), 4.45/5.13 (2d, 8H, monosubstituted cyclopentadienyl rings); ${ }^{13} \mathrm{C}-\mathrm{NMR}\left(\mathrm{CDCl}_{3}\right): \delta 162.68 / 133.32\left(\mathrm{C}_{4} / \mathrm{C}_{5}\right.$, selenadiazole rings), 69.37, 69.82 and 85.26 for the carbons of the monosubstituted cyclopentadienyl rings; $\mathrm{MS}(\mathrm{m} / \mathrm{z}, \%): 448\left(\mathrm{M}^{+}\right.$, $100 \%$ ); Anal. Calcd. For $\mathrm{C}_{14} \mathrm{H}_{10} \mathrm{~N}_{4} \mathrm{Se}_{2} \mathrm{Fe}$ : C, 37.53; H, 2.25; N, 12.50. Found: C, 37.51; H, 2.29; N, 12.41 .

General procedure for the preparation of 1,2,3-thiadiazole derivatives 15, 17, 19, 21 and 23 [13].

An excess amount of thionyl chloride was stirred at $0{ }^{\circ} \mathrm{C}$ and the hydrazones or semicarbazones $\mathbf{6}$, $7,8,10,11$ or 12 were added in several portions. The mixtures were stirred at room temperature overnight until no more hydrogen chloride was produced. The remaining thionyl chloride was evaporated under vacuum and the residue was washed with diethyl ether to give good yields of the corresponding 1,2,3-thiadiazoles as fine powders. A recrystallization from chloroform or dimethylsulfoxide was carried out when necessary.

4-Phenyl-[1,2,3]-thiadiazole (15). Beige powder, yield = 83\%; IR: $v$ 3094, 1605, 1456, 1408, 1281, 1068, $921 \mathrm{~cm}^{-1}$; ${ }^{1} \mathrm{H}-\mathrm{NMR}$ (DMSO-d ${ }_{6}$ ): $\delta$ 7.28-7.31 (m, 3H, aromatic), 7.53-7.55 (m, 2H, aromatic), $8.89\left(\mathrm{~s}, 1 \mathrm{H}\right.$, selenadiazole ring); ${ }^{13} \mathrm{C}-\mathrm{NMR}\left(\mathrm{DMSO}_{\mathrm{d}}\right)$ ) $\delta 126.81,127.42,127.90$ and 130.81 for benzene carbons, 161.31 and 134.60 for $\mathrm{C} 4$ and $\mathrm{C} 5$ carbons of the thiadiazole ring; MS (m/z, \%): 162 
$\left(\mathrm{M}^{+}, 100 \%\right)$; Anal. Calcd. For $\mathrm{C}_{8} \mathrm{H}_{6} \mathrm{~N}_{2} \mathrm{~S}$ : C, 59.24; H, 3.73; N, 17.27, S, 19.77. Found: C, 45.78; H, $2.93 ; \mathrm{N}, 13.49, \mathrm{~S}, 19.59$.

4-[1,2,3]-Thiadiazole-4-yl-phenol (17). Pale brown powder, yield = 76\%; IR: $\vee$ 3413, 3109, 1597, 1501, 1450, 1411, 1278, 1073, $927 \mathrm{~cm}^{-1} ;{ }^{1} \mathrm{H}-\mathrm{NMR}$ (DMSO-d ${ }_{6}$ ): $\delta 2.16$ (s, 1H, OH group), 7.21/7.56 (AA'BB', 4H, phenylene), 8.83 (s, 1H, thiadiazole ring); ${ }^{13} \mathrm{C}-\mathrm{NMR}$ (DMSO-d $\left.{ }_{6}\right): \delta 116.21 / 127.80(\mathrm{CH}$, phenylene), 130.61/159.11 (Cq, phenylene), 161.70/133.80 (C4/C5, thiadiazole ring carbons); MS (m/z, \%): $178\left(\mathrm{M}^{+}, 100 \%\right)$; Anal. Calcd. For $\mathrm{C}_{8} \mathrm{H}_{6} \mathrm{~N}_{2} \mathrm{OS}$ : C, 53.92; H, 3.39; N, 15.72, S, 17.99. Found: C, 53.71; H, 3.28; N, 15.61, S, 17.93 .

7,10,10-Trimethyl-3-thia-4,5-diaza-tricyclo[5.2.1.0 $\left.0^{2,6}\right]$ deca-2(6),4-diene (19). Pale yellow powder, yield $=58 \%$; IR: $v 2963,1609,1459,1406,1283,925 \mathrm{~cm}^{-1} ;{ }^{1} \mathrm{H}-\mathrm{NMR}\left(\mathrm{CDCl}_{3}\right): \delta 0.76\left(\mathrm{~s}, 3 \mathrm{H}\right.$ at $\left.\mathrm{C}_{9}\right)$, $0.93\left(\mathrm{~s}, 3 \mathrm{H}\right.$ at $\left.\mathrm{C}_{10}\right), 1.08\left(\mathrm{~s}, 3 \mathrm{H}\right.$ at $\mathrm{C}_{8}$ ), the peaks for the other protons were observed between (1.432.38); ${ }^{13} \mathrm{C}-\mathrm{NMR}\left(\mathrm{CDCl}_{3}\right): \delta 61.64\left(\mathrm{C}_{1}\right), 166.81\left(\mathrm{C}_{2}\right), 154.04\left(\mathrm{C}_{3}\right), 44.11\left(\mathrm{C}_{4}\right), 27.26\left(\mathrm{C}_{5}\right), 32.40\left(\mathrm{C}_{6}\right)$, $48.08\left(\mathrm{C}_{7}\right), 19.73\left(\mathrm{C}_{8}\right), 18.91\left(\mathrm{C}_{9}\right), 11.04\left(\mathrm{C}_{10}\right)$; $\mathrm{MS}(\mathrm{m} / \mathrm{z}, \%): 194\left(\mathrm{M}^{+}, 100 \%\right)$; Anal. Calcd. For $\mathrm{C}_{10} \mathrm{H}_{14} \mathrm{~N}_{2} \mathrm{~S}$ : C, 61.82; H, 7.26; N, 14.42. Found C, 61.85; H, 7.28; N, 14.46.

[1,2,3]-Thiadiazole-4-yl-ferrocene (21). Yellow powder, yield $=66 \%$; IR: $\vee$ 3068, 1608, 1458, 1413, 1274, $927 \mathrm{~cm}^{-1} ;{ }^{1} \mathrm{H}-\mathrm{NMR}\left(\mathrm{CDCl}_{3}\right): \delta 8.67\left(\mathrm{~s}, 1 \mathrm{H}\right.$, proton at $\mathrm{C}_{5}$ thiadiazole ring), $4.15(\mathrm{~s}, 5 \mathrm{H}$, unsubstituted cyclopentadienyl ring), 4.43/5.10 (2d, $4 \mathrm{H}$, monosubstituted cyclopentadienyl ring); ${ }^{13} \mathrm{C}$ NMR $\left(\mathrm{CDCl}_{3}\right): \delta 162.36$ and 133.49 for $\mathrm{C}_{4}$ and $\mathrm{C}_{5}$ thiadiazole ring, 68.12, 69.53, 69.68 and 84.89 for the carbons of the mono- and unsubstituted cyclopentadienyl rings; $\mathrm{MS}(\mathrm{m} / \mathrm{z}, \%): 270\left(\mathrm{M}^{+}, 100 \%\right)$; Anal. Calcd. For $\mathrm{C}_{12} \mathrm{H}_{10} \mathrm{~N}_{2} \mathrm{SFe}$ C, 53.36; H, 3.73; N, 10.37. Found: C, 53.61; H, 3.70; N, 10.42 .

Di-[1,2,3]-thiadiazole-4-yl-ferrocene (23). Yellow powder, yield $=61 \%$; IR: $\vee$ 3081, 1613, 1457, 1420, 1268, $926 \mathrm{~cm}^{-1} ;{ }^{1} \mathrm{H}-\mathrm{NMR}\left(\mathrm{CDCl}_{3}\right): \delta 8.72(\mathrm{~s}, 2 \mathrm{H}$, thiadiazole rings), 4.47/5.11 (2d, 8H, monosubstituted cyclopentadienyl rings); ${ }^{13} \mathrm{C}-\mathrm{NMR}\left(\mathrm{CDCl}_{3}\right): \delta 161.97 / 133.14\left(\mathrm{C}_{4} / \mathrm{C}_{5}\right.$, selenadiazole rings), 69.48, 69.71 and 84.99 for the carbons of the monosubstituted cyclopentadienyl rings; MS (m/z, \%): $354\left(\mathrm{M}^{+}, 100 \%\right)$; Anal. Calcd. For $\mathrm{C}_{14} \mathrm{H}_{10} \mathrm{~N}_{4} \mathrm{~S}_{2} \mathrm{Fe}: \mathrm{C}, 47.47$; H, 2.84; N, 15.82. Found: C, 47.56; H, 2.90; N, 15.84 .

\section{References}

1. Ando,W.; Tokitoh, N. Heteroatom Chem. 1991, 1.

2. Arsenyan, P.; Pudova, O.; Lukevics, E. Tetrahedron Lett. 2002, 43, 4817.

3. Regitz, M.; Krill, S. Phosphorus, Sulfur, Silicon, Relat. Elem. 1996, 99, 15.

4. Lalezari, I.; Shafiee, A. Tetrahedron Lett. 1969, 28, 5105.

5. Lalezari, I.; Shafiee, A. J. Org. Chem. 1971, 36, 2836.

6. Lalezari, I.; Shafiee, A.; Yalpani, M. J. Org. Chem. 1973, 38, 338.

7. Hurd, C. D.; Mori, R. I. J. Am. Chem. Soc. 1955, 77, 5359.

8. Detert, H.; Meier, H. Liebigs. Ann. 1997, 1557.

9. Zhon, Y.; Heimgartner, H. Helv. Chim. Acta 2000, 83, 539. 
10. Nishiyama, Y.; Hada, Y.; Anjiki, M.; Hanita, S.; Sonoda, N. Tetrahedron Lett. 1999, 40, 6293.

11. Arsenyan, P.; Oberte, K.; Pudova, O.; Lukevics, E. Chem. Heterocycl. Comp. 2002, 38, 1437.

12. Zimmer, O.; Meier, H. J. Chem. Soc, Chem. Commun. 1982, 481.

13. Hanold, N.; Kalbitz, H.; Al-Smadi, M.; Meier, H. Z. Naturforsch. 1995, 50b, 1121.

14. Curran, W. V.; Sassiver, M. L.; Boothe, J. H.; Jacob, L. J. Heterocycl. Chem. 1985, 22, 479.

15. Katritzky, A. R. Physical Methods in Heterocyclic Chemistry; John Wiley and Sons, Inc.: New York, 1963; vol. 2, p. 234.

16. Fujita, M.; Kobori, T.; Hiyama, T.; Kondo, K. Heterocycles 1993, 36, 33.

Sample availability: Available from the authors

(C) 2004 by MDPI (http://www.mdpi.org). Reproduction is permitted for noncommercial purposes. 